\title{
Semiconditional Electrical Stimulation of Pudendal Nerve Afferents Stimulation to Manage Neurogenic Detrusor Overactivity in Patients with Spinal Cord Injury
}

\author{
Young-Hee Lee, M.D., Ph.D., Jung Moon Kim, M.D., Hyung Tae Im, M.D., \\ Kye-Wook Lee, M.D., Sung Hoon Kim, M.D., Dong Min Hur, M.D.
}

Department of Rehabilitation Medicine, Yonsei University Wonju College of Medicine, Wonju 220-701, Korea

\begin{abstract}
Objective To evaluate the effect of semiconditional electrical stimulation of the pudendal nerve afferents for the neurogenic detrusor overactivity in patients with spinal cord injury. Forty patients (36 males, 4 males) with spinal cord injury who had urinary incontinence and frequency, as well as felt bladder contraction with bladder filling sense or autonomic dysreflexic symptom participated in this study.

Method Patients with neurogenic detrusor overactivity were subdivided into complete injury and incomplete injury groups by ASIA classification and subdivided into tetraplegia and paraplegia groups by neurologic level of injury. Bladder function, such as bladder volumes infused to the bladder until the first occurrence of neurogenic detrusor overactivity $\left(\mathrm{V}_{\mathrm{ini}}\right)$ and the last contraction suppressed by electrical stimulation $\left(\mathrm{V}_{\text {max }}\right)$ was measured by water cystometry (CMG) and compared with the results of each subgroup.

Results Among the 40 subjects, 35 patients showed neurogenic detrusor overactivity in the CMG study. Among these 35 patients, detrusor overactivity was suppressed effectively by pudendal nerve afferent electrical stimulation in 32 patients. The infusion volume until the occurrence of the first reflex contraction $\left(\mathrm{V}_{\text {ini }}\right)$ was $99.4 \pm 80.3 \mathrm{ml}$. The volume of saline infused to the bladder until the last contraction suppressed by semiconditional pudendal nerve stimulation $\left(\mathrm{V}_{\max }\right)$ was $274.3 \pm 93.2 \mathrm{ml}$, which was significantly greater than $\mathrm{V}_{\mathrm{ini}}$. In patients with good response to the pudendal nerve afferent stimulation, the bladder volume significantly increased by stimulation in all the patients.

Conclusion In this study, semiconditional electrical stimulation on the dorsal penile afferent nerve could effectively inhibit neurogenic detrusor overactivity and increase bladder volume in patients with spinal cord injury.
\end{abstract}

Key Words Spinal cord injuries, Detrusor overactivity, Pudendal nerve, Electrical stimulation 


\section{INTRODUCTION}

Neurogenic detrusor overactivity is defined as involuntary detrusor contraction induced due to a neurological condition during the filling phase of the bladder. ${ }^{1}$ When control function in the cerebrum and the pons is blocked due to injury of the spinal cord above the sacral cord, the bladder becomes overactive, resulting in urinary incontinence and frequency. ${ }^{2}$

Clean intermittent catheterization, parasympathetic blocking agents and other bladder muscle relaxants are used most frequently as the current treatment of choice for neurogenic detrusor overactivity in spinal cord injuries (SCI). The pharmacologic treatment method mentioned above resulted in a few side effects that were not tolerated by the patients. ${ }^{3}$ Another treatment method is botulinum toxin injection to the detrusor muscle, but this effect is not permanent. ${ }^{4}$ Surgical treatments, which expand bladder volume using the large intestine or other organs, can be applied with limitations, because of the possible risk of surgical complications and mucous production in the augmented bladder. ${ }^{5}$

Electrical stimulation of the sacral afferent nerve has been introduced as another treatment option for urinary incontinence caused in neurogenic detrusor overactivity. In 1992, Wheeler et al. ${ }^{6}$ reported cases where they expanded the bladder volume by suppression of neurogenic detrusor overactivity through the application of penile nerve stimulation, but the mechanism was not elucidated. Nonetheless, the pudendal pelvic reflex via local cord neurotransmission appeared to be the probable mechanism of inhibition. ${ }^{7,8}$ Electrical stimulation has advantages with its local effect, resulting in systemic side effects being lower than those caused by medication. It can also be controlled readily, consequently being used as one of methods of choice for treating urinary incontinence. ${ }^{9}$

For electrical stimulation to the pudendal nerve for the suppression of neurogenic detrusor overactivity in suprasacral spinal cord injury patients, two methods, continuous stimulation and conditional stimulation, have been introduced. ${ }^{10}$ The continuous method applies

A

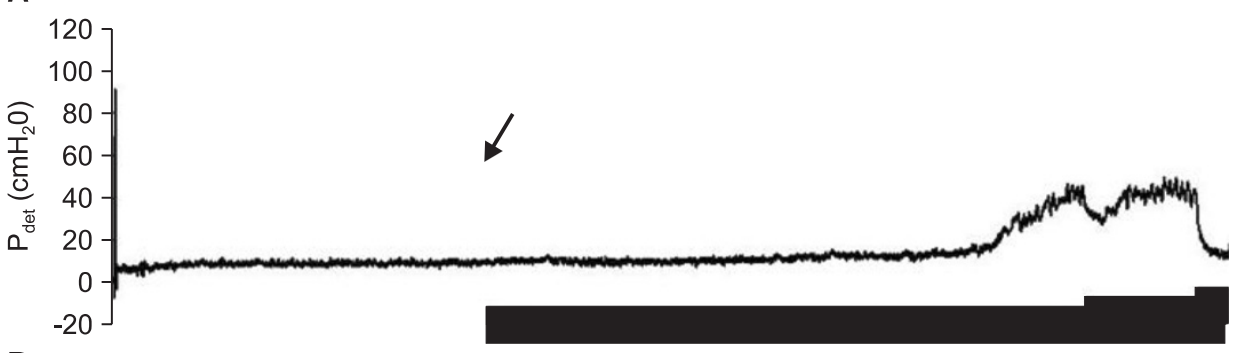

B

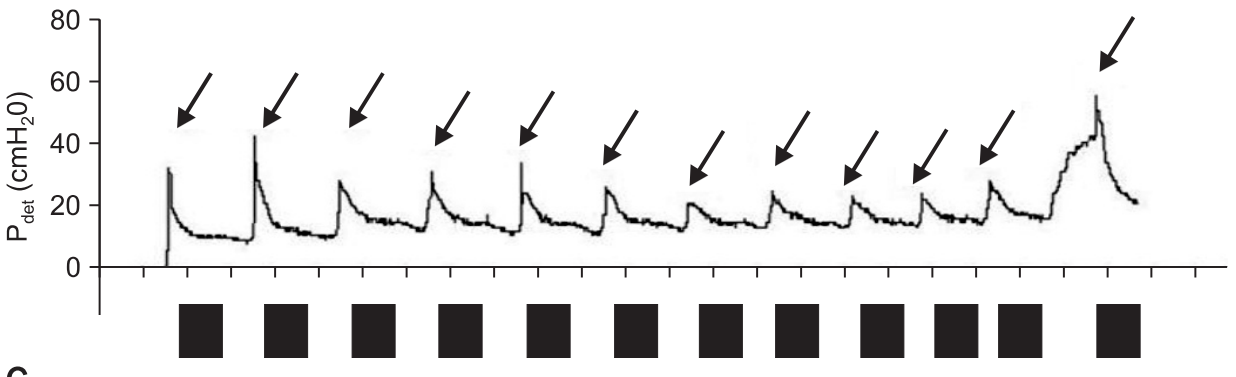

C

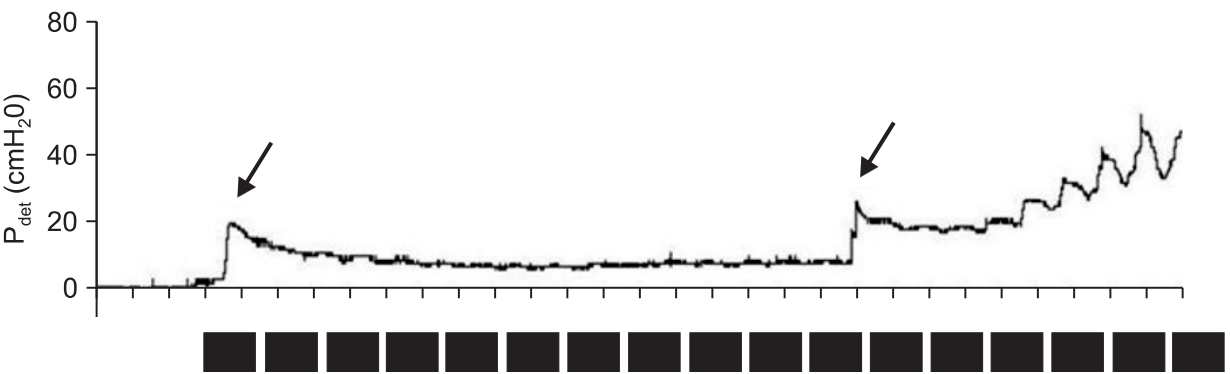

Fig. 1. CMG findings in different methods of pudendal afferent nerve electrical stimulation. Bold line represent the period of electrical stimulation. Arrow represents the switch control. (A) Continuous method applied the electrical stimulation throughout bladder filling. (B) Conditional method applied the electrical stimulation on each overactive contraction (C). Semiconditional stimulation started with the first bladder contraction and continued in a cyclic pattern with preset on-off time. The second arrow represents the manipulation of the equipment for intensity change. 
electrical stimulation throughout bladder filling (Fig. 1-A) whereas the conditional method initiates electrical stimulation as intravesical pressure begins to rise at the beginning of neurogenic detrusor overactivity (Fig. 1-B). Continuous stimulation may increase bladder volume readily and effectively, but it has shortcomings which include the lifespan of the battery and electrodes becoming shortened, as well as the effectiveness of electrical stimulation becoming less over time due to habituation.

When conditional stimulation is applied, patients have a method for detecting intravesical pressure such as fullness sensation and abdominal discomfort. Moreover, the stimulation setup in conditional stimulation is not suitable for the chronic setting because of battery consumption. ${ }^{10-12}$

To improve such shortcomings, we attempted a semiconditional stimulation that starts with the first bladder contraction, and continues in cyclic pattern with a preset on-off duration of burst of electric current (Fig. $1-\mathrm{C})$.

In this study, we examined whether semiconditional stimulation of the dorsal pudendal or clitoral nerve could prevent neurogenic detrusor overactivity and improve the storage function of the bladder in spinal cord injury patients of various levels of injury and severity of injury.

\section{Subjects}

Out of all the suprasacral spinal cord injury patients admitted to Wonju Christian Hospital from September 2002 to February 2007, this study was conducted on 40 patients ( 36 males and 4 females) that presented with frequent urination and urinary incontinence, and who felt bladder contraction and bladder fullness, or autonomic dysreflexia (AD) symptoms. This study was approved by the institutional review board (IRB).

The patients urinated every 4-6 hours by performing intermittent catheterization (IC) or applying reflex voiding, and presented with inconvenience of frequent urinary incontinence despite taking bladder muscle relaxants. The subjects were asked not to change their bladder management approach by using catheterization methods or bladder medications during their participation in this study. The mean age of the patients was 44 years (19-64 years), whereas the mean duration after injury was 8.2 years (1-19 years). Complete injury
Table 1. Subjects Compared by Neurologic Level of Injury and ASIA Classification

\begin{tabular}{lcccccc}
\hline & \multicolumn{2}{c}{ Tetraplegia } & \multicolumn{2}{c}{ Paraplegia } & \multicolumn{2}{c}{ Total } \\
\cline { 2 - 7 } & Male & Female & Male & Female & Male & Female \\
\hline Complete & 7 & 0 & 15 & 1 & 22 & 1 \\
Incomplete & 9 & 0 & 5 & 3 & 14 & 3 \\
Total & 16 & 0 & 20 & 4 & 36 & 4 \\
\hline
\end{tabular}

was observed in 23 patients and incomplete injury was in 17 patients. Sixteen patients were tetraplegics, whereas 24 were paraplegics (Table 1).

\section{MATERIALS AND METHODS}

\section{Urodynamic study}

Standard water cystometry (CMG) was performed in the supine position. 3-way transuretheral catheter was inserted in the bladder for pressure measurement, fluid infusion and ballooning. The pressure line was fluid filled and connected to the transducer in water cystometry Menuet $^{\mathrm{TM}}$ (Dantec, Skovlunde, Denmark). The balloon of the foley catheter was inflated to prevent leakage during the study. The initial fluid infusion rate was 30 $\mathrm{ml}$ per minute, and from the time of the onset of the first overactive contraction of the bladder, it was infused slowly at a rate closer to the natural filling rate of $10 \mathrm{ml}$ per minute.

In the cystometry test protocol using electrical stimulation, cystometry was stopped if; 1) The electrical stimulation to pudendal nerve did not suppress the reflex contraction; 2) The infusion volume was over $450 \mathrm{ml}$; and 3) the subject could not tolerate the test.

\section{Pudendal nerve afferent stimulation}

Electrical stimulation was delivered using the portable neuromuscular stimulator FOCUS ${ }^{\circledR}$ (Empi., Minnesota, USA) equipped with a remote switch through which the parameters could be programmed. A circular surface electrode $1 \mathrm{~cm}$ in diameter was used, and a cathode was placed in the proximal area of the dorsum of the penis at a $2 \mathrm{~cm}$ interval, and an anode was attached to the distal area. For female patients, a cathode was attached to the clitoris, and an anode was attached to the labium major. Biphasic rectangular pulses at $25 \mathrm{~Hz}$ frequency and 250 $\mu$ s pulse width were used. The current intensity was 


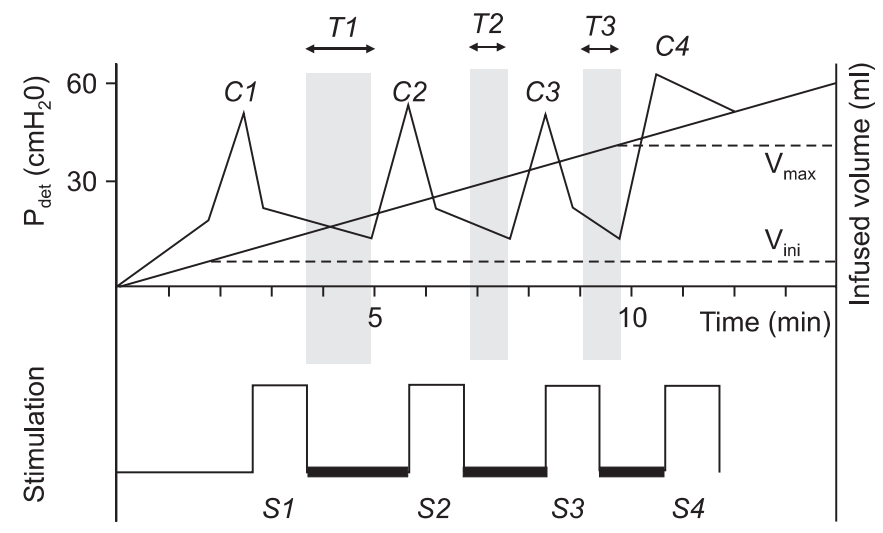

Fig. 2. Schematic representation of CMG with electrical stimulation to the pudendal nerve. $C 1, C 2 \ldots C n$ : Overactive bladder contraction. S1, S2...Sn: 50 seconds electrical stimulation to pudendal nerve at each overactive bladder contraction. T1, T2...Tn: Duration of suppression after stimulation at each overactive contraction. Duration measured from the end of the burst to the start of the next contraction. $V_{\text {ini: }}$ The infusion volume until the occurrence of the first overactive contraction. $\mathrm{V}_{\max }$ : The infusion volume to the bladder until the last contraction suppressed by semiconditional pudendal nerve stimulation.

set between 20 and $60 \mathrm{~mA}$ at a level equal to twice the threshold for reflex contraction of anal sphincter.

\section{Programming for Semiconditional electrical stimulation}

Semiconditional stimulation was the programmed current delivery which started with the first bladder contraction, and continued in cyclic pattern with preset on-off duration (Fig. 1-C). When the elevation of bladder pressure showing typical overactive contraction was detected during second CMG, transcutaneous dorsal penile-clitoral nerve stimulation was performed for 50 seconds (Fig. 1-B), which was sufficient to lower detrusor pressure to a pre-contraction level. ${ }^{13}$

To determined the efficient 'off' duration, the period of suppression (T1, T2, T3 in Fig. 2) by 50 seconds pudendal nerve stimulation was identified by measuring the interval from the end of stimulation to the occurrence of the following reflexic contraction. Switch-on duration of cyclic stimulation was 50 seconds, and switch-off duration was determined as 5 seconds shorter than the shortest duration of suppression (T3 in Fig. 2) by 50 seconds stimulation during second CMG.

\section{Protocol for cystometrography}

The CMG was performed a total of 3 times during the test. The first CMG was performed to determine whether the experimental group had overactive contraction and to assess the bladder volume. The test was not preceded if there was no overactive detrusor contraction. The second CMG was performed to see whether neurogenic detrusor overactivity was effectively suppressed, and to assess the duration of the suppression by 50 seconds pudendal nerve afferent stimulation. The third CMG was performed to assess the effect of semiconditional stimulation, which started with the first bladder contraction, and continued in a cyclic pattern with preset on-off duration determined by the second CMG results. There were at least 30 minute intervals between each CMG.

In the third CMG, the volume of saline volume infused to the bladder until the first occurrence of overactive contraction $\left(\mathrm{V}_{\text {ini }}\right)$ and the volume of saline infused to the bladder until the last contraction suppressed by electric stimulation $\left(\mathrm{V}_{\max }\right)$ were compared and analyzed to determine whether the four different groups of spinal cord lesion were different. For statistical analysis, paired t-test was applied, and cases with $\mathrm{p}<0.01$ were determined to be significant.

\section{RESULTS}

Out of the 40 patients who felt voiding desire in response to fullness or AD symptoms, typical neurogenic detrusor overactivity did not occur in 5 patients in the first CMG. Of the 35 patients with neurogenic detrusor overactivity, overactive contraction was effectively suppressed by dorsal penile-clitoral nerve stimulation in 32 patients.

Out of 31 male patients with neurogenic detrusor overactivity, dorsal penile nerve stimulation was not effective in only 1 patient. Out of 4 female patients, dorsal clitoral nerve stimulation suppressed bladder contraction effectively in just 2 patients (Table 2).

Fig. 3 demonstrates the results of semiconditional stimulation with efficient on-off duration from one of the subjects who showed effective suppression of overactive bladder by pudendal nerve stimulation.

In patients with detrusor overactivity, which was suppressed effectively by semiconditional pudendal nerve stimulation, the infusion volume until the occurrence of the first reflex contraction $\left(\mathrm{V}_{\mathrm{ini}}\right)$ was 
Table 2. Results of Pudendal Nerve Afferent Stimulation for Detrusor Overactivity in Patients with Spinal Cord Injury, According to the Severity and Level of Injury

\begin{tabular}{|c|c|c|c|c|c|c|}
\hline \multirow{2}{*}{$\begin{array}{l}\text { Detrusor } \\
\text { overactivity } \\
\text { (number) }\end{array}$} & \multirow{2}{*}{$\begin{array}{c}\text { Suppression by } \\
\text { Electrical Stimulation } \\
\text { (number) }\end{array}$} & \multirow{2}{*}{$\begin{array}{c}\text { Sex } \\
\text { (number) }\end{array}$} & \multirow{2}{*}{ Severity* } & \multicolumn{2}{|c|}{ Level } & \multirow{2}{*}{ Sum } \\
\hline & & & & Tetra $^{\dagger}$ & Para $^{*}$ & \\
\hline \multirow[t]{4}{*}{ No (5) } & & Male (5) & Com & 2 & 2 & 4 \\
\hline & & & Incom & 1 & & 1 \\
\hline & & Female (0) & Com & & & 0 \\
\hline & & & Incom & & & 0 \\
\hline \multirow[t]{9}{*}{ Yes (35) } & Yes (32) & Male (30) & Com & 5 & 13 & 18 \\
\hline & & & Incom & 7 & 5 & 12 \\
\hline & & Female (2) & Com & 0 & 0 & 0 \\
\hline & & & Incom & 0 & 2 & 2 \\
\hline & No (3) & Male (1) & Com & 0 & 0 & 0 \\
\hline & & & Incom & 1 & 0 & 1 \\
\hline & & Female (2) & Com & 0 & 1 & 1 \\
\hline & & & Incom & 0 & 1 & 1 \\
\hline & Total & & & 16 & 24 & 40 \\
\hline
\end{tabular}

Com: Complete, Incom: Incomplete

*Severity is defined by American Spinal cord Injury Association scale. ${ }^{\dagger}$ Tetra: Tetraplegia, ${ }^{\ddagger}$ Para: Paraplegia

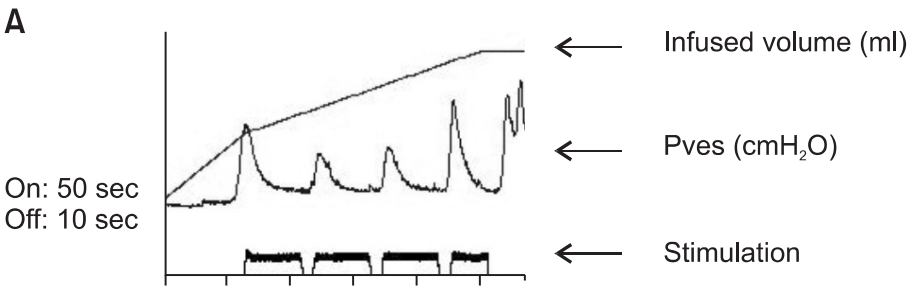

B

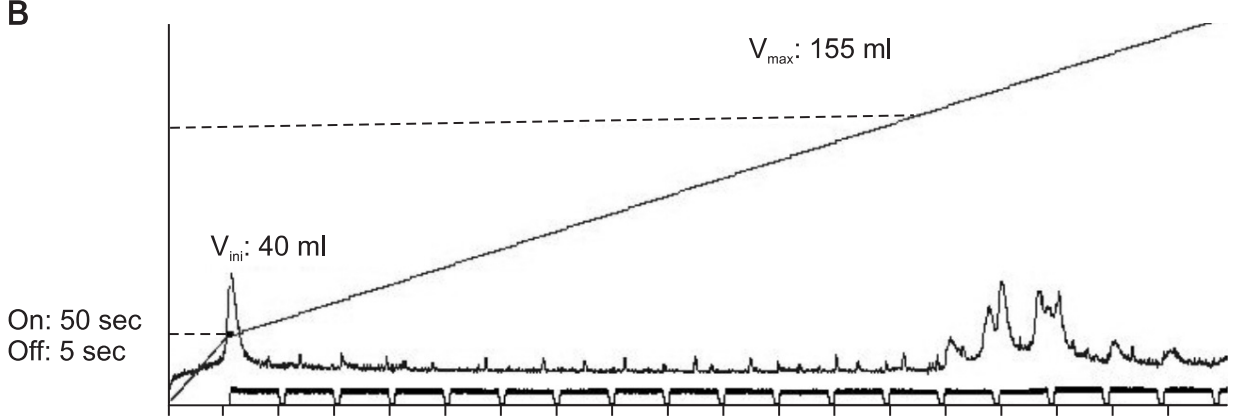

Fig. 3. CMG findings of semiconditional pudendal nerve stimulation to neurogenic detrusor overactivity. (A) When the switch-off duration was set to 10 seconds after 50 second burst of the current, overactive contractions suppressed only during 'on' period and occurred during every 'off' period. (B) When the switch-off duration was shortened to 5 seconds in the same patient, overactive detrusor contractions were effectively suppressed continuously until the infused volume reached approximately 4 times of $\mathrm{V}_{\text {ini. }}$
$99.4 \pm 80.3 \mathrm{ml} . \mathrm{V}_{\max }$, the volume of saline injected to the bladder until the last contraction suppressed by semiconditional pudendal nerve stimulation was $274.3 \pm 93.2 \mathrm{ml}$, which was significantly greater than $\mathrm{V}_{\mathrm{ini}}$ (p-value $<0.01$ ).

The suppressive effect of pudendal nerve afferent stimulation to detrusor overactivity was compared according to the level and severity of spinal cord injury. $\mathrm{V}_{\text {ini }}$ of the complete injury group and the incomplete injury group were $102.2 \pm 78.6 \mathrm{ml}$, and $105.9 \pm 98.1 \mathrm{ml}$, respectively, which was not statistically different ( $\mathrm{p}$ $<0.01$ ). $\mathrm{V}_{\max }$ of the complete injury group was $261.1 \pm 90.2$ $\mathrm{ml}$, which was significantly greater than $\mathrm{V}_{\text {ini }}$ of the same group $(p<0.01) . V_{\max }$ of the incomplete injury group 
Table 3. Bladder Volume During CMG with Semiconditional Electrical Stimulation to the Pudendal Nerve by Severity of Spinal Cord Injury

\begin{tabular}{lccc}
\hline & Complete & Incomplete & p-value \\
\hline $\mathrm{V}_{\text {ini }}{ }^{*}$ & $102.2 \pm 78.6$ & $105.9 \pm 98.1$ & $>0.01$ \\
$\mathrm{~V}_{\max }{ }^{\dagger}$ & $261.1 \pm 90.2$ & $294.4 \pm 92.6$ & $>0.01$ \\
(difference) & $(158.8 \pm 14.9)$ & $(188.5 \pm 20.1)$ & \\
p-value & $<0.01$ & $<0.01$ & \\
\hline
\end{tabular}

${ }^{*} \mathrm{~V}_{\mathrm{ini}}$ : The infusion volume until the occurrence of the first overactive contraction, ${ }^{\dagger} \mathrm{V}_{\text {max }}$ : The infusion volume to the bladder until the last contraction suppressed by semiconditional pudendal nerve stimulation

Table 4. Bladder Volume During CMG with Semiconditional Electrical Stimulation to the Pudendal Nerve by Neurological Level of Spinal Cord Injury

\begin{tabular}{lccc}
\hline & Tetraplegia & Paraplegia & p-value \\
\hline $\mathrm{V}_{\text {ini }}{ }^{*}$ & $89.3 \pm 78.7$ & $112.6 \pm 91.2$ & $>0.01$ \\
$\mathrm{~V}_{\text {max }}{ }^{\dagger}$ & $280.8 \pm 75.6$ & $272.6 \pm 101.3$ & $>0.01$ \\
(difference) & $(160 \pm 15.8)$ & $(191.5 \pm 18.5)$ & \\
p-value & $<0.01$ & $<0.01$ & \\
\hline
\end{tabular}

${ }^{*} \mathrm{~V}_{\text {ini: }}$ : The infusion volume until the occurrence of the first overactive contraction, ${ }^{\dagger} \mathrm{V}_{\text {max }}$ : The infusion volume to the bladder until the last contraction suppressed by semiconditional pudendal nerve stimulation

was $294.4 \pm 92.6 \mathrm{ml}$, which also significantly increased $(\mathrm{p}<0.01) . \mathrm{V}_{\max }$ of the incomplete and complete injury group was not significantly different (Table 3 ).

Experiment results were compared by dividing the subjects to tetraplegia and paraplegia according to their neurological level. $\mathrm{V}_{\mathrm{ini}}$ of the tetraplegia and paraplegia group was $89.3 \pm 78.7 \mathrm{ml}$ and $112.6 \pm 91.2 \mathrm{ml}$, respectively, which was not significantly different. $\mathrm{V}_{\max }$ of the tetraplegia group was $280.8 \pm 75.6 \mathrm{ml}$, the paraplegia group was $272.6 \pm 101.3 \mathrm{ml}$, and the bladder volume significantly increased by semiconditional pudendal nerve afferent stimulation in both groups $(\mathrm{p}<0.01)$ (Table 4).

\section{DISCUSSION}

The purpose of neurogenic bladder management in spinal cord injury patients is to preserve renal function and maintain urinary continence, consequently improving routine life or social activity. Neurogenic detrusor overactivity appears frequently in suprasacral spinal cord injury patients and is closely associated with low compliance, vesicoureteral reflux, bladder wall deformity, and renal injury. Therefore, treatments for neurogenic detrusor overactivity are very important.

Studies on electrical stimulation for the suppression of neurogenic detrusor overactivity that appeared in spinal cord injury patients could be divided to two groups. The first group comprised of treatment methods applied to acute urinary incontinence in non-spinal injury patients, on the delayed effect of electrical stimulation, and the treatment effect was anticipated by performing the stimulation for several hours daily for a certain period. Ishigooka et al. reported that in spinal cord injury patients, percutaneous stimulation was applied to the pelvic muscles and the $3^{\text {rd }}$ sacral foramina, and the patients were treated for several hours per day for 4 weeks. Improvement of symptoms was observed in 66-75\%, but cases with complete disappearance of urinary incontinence were less than $20 \% .^{14,15}$ The second group comprised of studies on immediate suppression effects on neurogenic detrusor overactivity during electrical stimulation. Wheeler et al. ${ }^{6}$ and Previnaire et al. ${ }^{16}$ reported that while supraspinal cord injury men underwent penile nerve electrical stimulation testing with water cystometry, overactive contraction did not occur, and thus a significantly larger volume could be injected.

In regard to the mechanism of immediate effects, reports showed that electrical stimulation of the pudendal afferent nerve suppressed the neurogenic detrusor overactivity immediately through the pudendal-hypogastric and pudendal-pelvic spinal cord reflex pathway. ${ }^{17}$ The mechanism of delayed effects was explained as the plasticity of the nervous control function of the central nervous system. ${ }^{18}$ In clinical studies for the suppression of neurogenic detrusor overactivity contraction, application of immediate effects is more effective.

Lee et al. ${ }^{13,19}$ reported that the electrical stimulation of the pudendal nerve afferent suppressed the neurogenic detrusor overactive contractions effectively on spinal cord injury of diverse severities and levels, and pudendal afferent nerve electrical stimulation could be used not only in laboratories but also at home. Kirkham 
et al. ${ }^{10}$ compared the effect of the suppression of neurogenic detrusor overactivity by dorsal penile nerve stimulation, which was performed either continuously or conditionally in the identical subjects, and reported that conditional stimulation was more effective. Nonetheless, for conditional stimulation, a switch has to be pushed whenever reflex contraction occurs. Hence, the patient must pay attention to the occurrence of reflex contraction, resulting in lower compliance of patients.

In our study, we applied semiconditional stimulation to improve the above mentioned limitation. If patients turned the switch on when they felt bladder contraction for the first time, the cyclic burst currents with preset on-off duration determined by the CMG finding was delivered. Manipulation of the equipment is required only for increasing the intensity of the current, or turning the switch off. This mode needs less attention than conditional stimulation, and saves the battery life compared to continuous stimulation

The 4 female patients showed unfavorable results compared to the male patients. Neurogenic detrusor overactivity was effectively suppressed by the dorsal nerve during clitoris stimulation in only 2 patients (50 $\%$ ). Although only one of two patients showed a positive response, the bladder volume increased effectively from $280 \mathrm{ml}\left(\mathrm{V}_{\text {ini }}\right)$ to $450 \mathrm{ml}\left(\mathrm{V}_{\max }\right)$, and $60 \mathrm{ml}$ to $96 \mathrm{ml}$ for the other patient. They were also disadvantages in applying electrical stimulation, such as difficulty of attaching electrodes or keeping it dry.

One possible side effect of pudendal nerve afferent stimulation is autonomic dysreflexia (AD). Although no cases showed AD provoked by electrical stimulation during this study, Lee et al. reported dorsal penile nerve stimulation during overactive bladder lowered elevated blood pressure, ${ }^{20}$ so possible development of AD should be considered.

The type of abnormalities in the lower urinary tract might be different in spinal cord injury patients with same neurological deficits. Also, non-neurogenic factors such as gender, personality, financial level, level of family support, or social activity should be considered for planning optimal management methods of the voiding problem. Therefore, the management of neurogenic bladder should be individualized to help each patient effectively, and should be performed by designing tests suitable to the special needs of each patient.
For the management of voiding dysfunction in suprasacral spinal cord injury patients, electrical stimulation treatment may be applied as an alternative to other methods, such as drug treatment or surgery. In our study, semiconditional electrical stimulation to the pudendal nerve effectively suppressed unwanted bladder contraction and increased bladder volume in 32 of 40 subjects $(80 \%)$. If spinal cord injury patients could be aware of bladder contraction, this method can be easy and safe, and suppress unnecessary overactive detrusor contraction.

One of the limitations of our study was performing 3 consecutive CMGs with 30 minute intervals in a day. Sequential CMGs could affect responses of the detrusor muscle. ${ }^{21}$ Also delayed effect of semiconditional stimulation to the pudendal nerve and results of the long-term trial should be studied carefully for further clinical application of our results. During this study, we studied sequential CMGs, which affected the response of the detrusor muscle.

In future studies, the treatment protocol of applying semiconditional stimulation to the pudendal nerve will be used for a prolonged period at home, and clinical findings, including voiding pattern and feasibility will be compared.

\section{CONCLUSION}

In this study, semiconditional electrical stimulation on dorsal penile afferent nerve could inhibit the neurogenic detrusor overactivity and increase the bladder volume in SCI patients with variable level and severity, effectively.

\section{ACKNOWLEDGEMENTS}

This work was supported by a research grant from Institute of Lifestyle Medicine.

\section{REFERENCES}

1. Abrams P, Cardozo L, Fall M, Griffiths D, Rosier P, Ulmsten U, Van Kerrebroeck P, Victor A, Wein A. The standardisation of terminology in lower urinary tract function: report from the standardisation subcommittee of the International Continence Society. Urology 2003; 61: 37-49 
2. Perkash I. Long-term urologic management of the patient with spinal cord injury. Urol Clin North Am 1993; 20: 423-434

3. Wein AJ. Pharmacologic options for the overactive bladder. Urology 1998; 51: 43-47

4. Sahai A, Khan MS, Gregson N, Smith K, Dasgupta P. Botulinum toxin for detrusor overactivity and symptoms of overactive bladder: where we are now and where we are going. Nat Clin Pract Urol 2007; 4: 379-386

5. Vignes JR, Gimber E, Sesay M, De Seze M, Guerin J, Joseph PA. Treatment of overactive bladder. ISS 2004; 7: 163-174

6. Wheeler JS Jr, Walter JS, Zaszczurynski PJ. Bladder inhibition by penile nerve stimulation in spinal cord injury patients. J Urol 1992; 147: 100-103

7. Fall M, Lindstrom S. Electrical stimulation. A physiologic approach to the treatment of urinary incontinence. Urol Clin North Am 1991; 18: 393-407

8. Yoshimura N. Bladder afferent pathway and spinal cord injury: possible mechanisms inducing hyperreflexia of the urinary bladder. Prog Neurobiol 1999; 57: 583-606

9. Schmidt RA, Jonas U, Oleson KA, Janknegt RA, Hassouna MM, Siegel SW, van Kerrebroeck PE. Sacral nerve stimulation for treatment of refractory urinary urge incontinence. Sacral Nerve Stimulation Study Group. J Urol 1999; 162: 352-357

10. Kirkham AP, Shah NC, Knight SL, Shah PJ, Craggs MD. The acute effects of continuous and conditional neuromodulation on the bladder in spinal cord injury. Spinal Cord 2001; 39: 420-428

11. Dalmose AL, Rijkhoff NJ, Kirkeby HJ, Nohr M, Sinkjaer T, Djurhuus JC. Conditional stimulation of the dorsal penile/clitoral nerve may increase cystometric capacity in patients with spinal cord injury. Neurourol Urodyn 2003; 22: 130-137

12. Hansen J, Media S, Nohr M, Biering-Sorensen F, Sinkjaer T, Rijkhoff NJ. Treatment of neurogenic detrusor overactivity in spinal cord injured patients by conditional electrical stimulation. J Urol 2005; 173: 2035-2039
13. Lee JM, Lee YH, Lee SS, Jang SM, Song JM, Song KH. Inhibition of Hyper-reflexic detrusor contraction by sacral afferent nerve stimulation in spinal cord injury. J Korean Acad Rehab Med 2001;25: 956-963

14. Ishigooka M, Suzuki Y, Hashimoto T, Sasagawa I, Nakada T, Handa Y. A new technique for sacral nerve stimulation: a percutaneous method for urinary incontinence caused by spinal cord injury. Br J Urol 1998; 81: 315-318

15. Ishigooka M, Hashimoto T, Hayami S, Suzuki Y, Nakada T, Handa Y. Electrical pelvic floor stimulation: a possible alternative treatment for reflex urinary incontinence in patients with spinal cord injury. Spinal Cord 1996; 34: 411-415

16. Previnaire JG, Soler JM, Perrigot M, Boileau G, Delahaye H, Schumacker P, Vanvelcenaher J, Vanhée JL. Short-term effect of pudendal nerve electrical stimulation on detrusor hyperreflexia in spinal cord injury patients: importance of current strength. Paraplegia 1996; 34: 95-99

17. Lindstrom S, Fall M, Carlsson CA, Erlandson BE. The neurophysiological basis of bladder inhibition in response to intravaginal electrical stimulation. J Urol 1983; 129: 405-410

18. Tanagho EA, Schmidt RA. Electrical stimulation in the clinical management of the neurogenic bladder. J Urol 1988; 140: 1331-1339

19. Lee YH, Creasey GH. Self-controlled dorsal penile nerve stimulation to inhibit bladder hyperreflexia in incomplete spinal cord injury: a case report. Arch Phys Med Rehabil 2002; 83: 273-277

20. Lee YH, Creasey GH, Lim H, Song J, Song K, Kim J. Detrusor and blood pressure responses to dorsal penile nerve stimulation during hyperreflexic contraction of the bladder in patients with cervical cord injury. Arch Phys Med Rehabil 2003; 84: 136-140

21. Ockrim J, Laniado ME, Khoubehi B, Renzetti R, Agro EF, Carter SS, Tubaro A. Variability of detrusor overactivity on repeated filling cystometry in men with urge symptoms: comparison with spinal cord injury patients. BJU Int 2005; 95: 587-590 